\title{
A Coupled System for Investigating the Physics of Wave-Ice Interactions
}

\author{
MARK D. ORZECH \\ U.S. Naval Research Laboratory, Stennis Space Center, Mississippi \\ FENGYAN SHI \\ Center for Applied Coastal Research, University of Delaware, Newark, Delaware \\ JAyARAm VeERAmony, SAmuel BATEMAn, AND Joseph CALANTONi \\ U.S. Naval Research Laboratory, Stennis Space Center, Mississippi \\ JAMES T. KIRBY \\ Center for Applied Coastal Research, University of Delaware, Newark, Delaware
}

(Manuscript received 2 November 2017, in final form 22 March 2018)

\begin{abstract}
A coupled model system has been developed to investigate the physics of wave attenuation and ice edge retreat in the marginal ice zone (MIZ) at small scales $[O(\mathrm{~m})]$. A phase-dependent finite-volume/finitedifference fluid dynamics model is used to simulate waves and currents, and a discrete element software package is employed to represent ice floes as bonded collections of individually tracked smaller particles. We first review the development of the coupled system, with an emphasis on the coupling software and the representation of wave-ice shear stress. Then we describe a series of simulations that were conducted to evaluate and qualitatively validate the performance of the coupled models. The system produced reasonable results for cases of a vertically oscillating ice block and a free-floating ice floe in monochromatic waves. In larger-scale simulations involving multiple ice floes and pancake ice, estimated transmission and reflection coefficients were similar to those obtained from alternate models and/or data, although numerical dissipation may have reduced estimates of transmitted wave energy in longer wave flumes. Challenges and limitations involving relative length scales in the coupled wave and ice domains are explained and discussed.
\end{abstract}

\section{Introduction}

As the effects of climate change continue to reshape the polar regions and reduce the ice cover, larger areas of open ocean allow for the growth of more energetic surface waves than have previously been seen (Thomson and Rogers 2014). The larger waves are playing an increasingly important role in breaking up the ice edge and the floes in marginal ice zones (MIZs) along pack ice boundaries. In seeking to better understand and predict the processes and evolving properties of this dynamic zone, ocean modelers have taken a range of different approaches.

Corresponding author: Mark Orzech, mark.orzech@nrlssc. navy.mil
Most operational modeling of wave-ice interaction is at scales of $O(\mathrm{~km})$ or larger, with the effects of individual waves and floes represented statistically or parametrically. Multiple methods have been developed to estimate the effects of MIZ ice floes on ocean waves (e.g., Squire et al. 1995; Wadhams 1986; Liu et al. 1992; Keller 1998). The "mass loading" approach (Wadhams and Holt 1991) modifies wave velocity by altering the real part of the wavenumber $k_{r}$ and may be used in most areas of the MIZ; however, it does not estimate wave attenuation and thus is of limited applicability. The "surface layer" approach (e.g., Liu et al. 1993) applies to solid shore-fast or pack ice, assuming that the wave dissipation is caused by viscosity in the turbulent boundary layer beneath the ice. The "floe scattering" approach (e.g., Kohout and Meylan 2008; Dumont et al. 2011) is 
designed for scenarios with less concentrated floes, near the outer edge of the MIZ. The "viscous layer" approach (e.g., Keller 1998; Wang and Shen 2010; Zhao and Shen 2015) applies to more highly concentrated floe fields, assigning the ice layer a rheology with specific viscoelastic properties similar to a dense fluid. In general, the parametric models are limited to tuning their coefficients in order to better match available data. They cannot achieve a fully physical representation of ice floe behavior at small scales.

Other modeling efforts have focused on a more physically accurate representation of the ice and its behavior in response to waves and currents. Chief among these are models that simulate ice and floes as flexible thin plates, or as collections of bonded elements using the discrete element method (DEM). The thin-elasticplate approach is commonly used in combination with wave models based on linear potential flow theory (Bennetts et al. 2010; Peter and Meylan 2010; Kohout and Meylan 2008; Toffoli et al. 2015) and has been effective at estimating wave dissipation by large fields of irregular thin-plate floes. However, this approach provides no information about the effects of waves on the ice floes and cannot anticipate changes to its floe field without relying on other models or data sources.

Recent experimental and field results also suggest that there are several areas where the combination of thin plates and linear theory fails to capture all relevant wave-ice interaction processes. Laboratory measurements using tethered plates in a wave flume produce greater attenuation than predicted by thin-plate/lineartheory models, particularly for steeper waves (Bennetts et al. 2015; Toffoli et al. 2015). The authors suggest that this attenuation is at least partly due to additional wave dissipation caused by wave overwash of the floating plates, as well as by collisions of neighboring plates when ice density is high. In addition, field measurement results (Kohout et al. 2014; Meylan et al. 2014) indicate that there may be greater penetration of higher energy waves into the MIZ and pack ice than was previously predicted by the existing models.

With a DEM representation of ice floes, each element corresponds to a small ice particle that is individually tracked and bonded to neighboring elements to constitute a floe or a larger ice layer. The formation, evolution, motion, and interaction of ice floes can thus be simulated over scales ranging from $O(\mathrm{~m})$ to $O(1000) \mathrm{km}$ or more, depending on the element size selected and the availability of computational resources. Earlier models (e.g., Hopkins and Shen 2001; Hopkins and Thorndike 2006) represent each complete ice floe as a single element of $O(100-1000) \mathrm{m}$ and are applied to larger domains, such as Cook Inlet, Alaska. More recent DEM efforts (e.g., Xu et al. 2012; Polojärvi and Tuhkuri 2013; Herman 2013, 2017; Orzech et al. 2014; Song et al. 2014) utilize collections of smaller bonded elements $[O(\mathrm{~cm}-\mathrm{m})]$ to represent floes or sections of ice. While in general more expensive computationally, this approach allows for investigation of smaller-scale ice floe material properties and behavior in response to wave forcing.

The coupled wave-ice system described here follows the latter, small-scale approach, representing both waves and ice at resolutions of $O(\mathrm{~m})$ in domains of $O(1-10) \mathrm{km}^{2}$. Waves are simulated with the NonHydrostatic WAVE model (NHWAVE; Ma et al. 2012, 2016; Derakhti et al. 2015), and ice floes are represented using the DEM package Large-Scale Atomic/ Molecular Massively Parallel Simulator (LAMMPS) Improved for General Granular and Granular Heat Transfer Simulations (LIGGGHTS; Kloss et al. 2012). The transfer of energy and momentum between waves and floes is tracked throughout the wave-ice domain by both models (see also Orzech et al. 2016b; Bateman et al. 2016; Orzech et al. 2014). The system is designed to resolve waves at kilometer scales, accurately simulating their evolution and attenuation when passing through a field of ice floes that realistically flex, collide, and fracture. In general, the vertical thickness of the ice floes $[O(\mathrm{~m})]$ is small relative to their horizontal dimensions $[O(100) \mathrm{m}-\mathrm{km}]$, and individual elements must be small relative to all floe dimensions.

The advantage of such a small-scale system is that it can be more fully grounded in the basic physics of waves and ice, in contrast to larger regional and global ocean models in which both wave and ice effects are generally represented with empirical parameterizations. The small-scale approach facilitates the investigation of fundamental scientific questions, such as the role of interfloe collisions and floe fracturing in wave attenuation, the effects of increasing wave energy levels on the composition and floe size distribution (FSD) of a given marginal ice zone, and the implications of increasing temperatures and weakening ice for the future of the polar ice cover. By confirming that the assumptions of selected large-scale parameterizations are consistent with the relevant small-scale physics, this coupled waveice system can provide a more solid grounding and validation for those broader approximations. NHWAVE is well suited for incorporating surface objects, as its vertical $\sigma$ coordinate can be mapped directly onto object surfaces (see below), and it is more efficient than other models with uniform vertical grid spacing (Ma et al. 2012; Derakhti et al. 2015). In contrast to existing thin-plate or viscoelastic ice floe representations, the LIGGGHTS discrete element system allows individual 


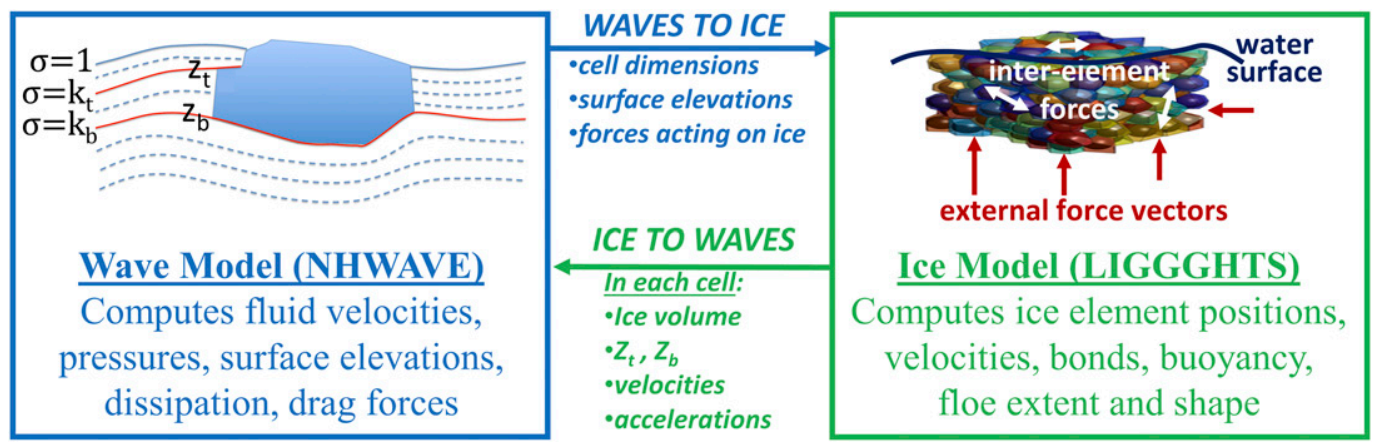

FIG. 1. Wave-ice system components and coupling communication. Image in each box illustrates that model's perspective on the wave-ice problem. In the wave model domain, $\sigma$ levels for surface $(=1)$, top $(t)$, and bottom $(b)$ of floe are indicated. Terms $z_{t}$ and $z_{b}$ represent the top and bottom elevations of ice in each cell, respectively, computed in LIGGGHTS and passed to NHWAVE.

ice particles and bonds to be tracked so that stresses, strains, and fracturing within each ice floe can be quantitatively measured and mapped in time and space. Coupled together, the two models may be used to monitor the total energy and momentum balances for waves passing through a marginal ice zone, identifying primary and secondary processes that affect wave attenuation and ice edge retreat.

Below we summarize the development of the coupled wave-ice system and present results from a series of tests designed to evaluate general system performance. While the coupled system simulates the internal properties of ice, the tests described herein were primarily focused on how the motion of solid ice blocks was affected by interacting with the fluid and how the fluid pressure and fluxes were altered by the floating ice. An overview of the NHWAVE and LIGGGHTS models is presented in section 2, including a description of coupling software developed to enable communication between the two system components and the implementation of drag forces. Model evaluation tests and qualitative validations are described in section 3 , followed by additional discussion and conclusions in section 4 .

\section{Coupled model development}

In its present state, the coupled wave-ice system (Fig. 1) consists of a nonhydrostatic, fully dispersive surface wave model (NHWAVE) linked to a particletracking code in which ice floes are represented as collections of bonded discrete elements (LIGGGHTS). The integrated coupling software allows relevant physical data, such as fluid pressure, fluid velocities, floe shape, and floe position, to be passed between the two model domains. Initially, each model required significant modifications to facilitate the wave-ice coupling.
NHWAVE was adapted to accommodate floating surface objects, accounting for the effects of their motion and acceleration as well as determining surface and form drag. LIGGGHTS was configured to compute and track bonds between ice elements and to follow specific criteria for the fracture of the bonds, accepting forcing vectors, surface elevations, and other domain-related information from the wave model. As many of these developments are detailed in Orzech et al. (2016a; see, in particular, their Figs. 1-3) and Bateman et al. (2018, manuscript submitted to Mech. Res. Commun.), they will only be summarized in the following three subsections.

\section{a. Wave model}

NHWAVE solves the incompressible Navier-Stokes equations in a 3D domain, with a surface-/bed-following $\sigma$ coordinate in the vertical direction and Cartesian axes in the horizontal $x$ and $y$ directions. In fully Cartesian form, these equations may be written as

$$
\begin{aligned}
\frac{\partial u_{i}}{\partial x_{i}} & =0 \\
\frac{\partial u_{i}}{\partial t}+u_{j} \frac{\partial u_{i}}{\partial x_{j}} & =-\frac{1}{\rho} \frac{\partial P}{\partial x_{i}}+g_{i}+\frac{\partial \tau_{i j}}{\partial x_{j}},
\end{aligned}
$$

where $i$ and $j$ each represent the three dimensions, with, for example, $\left(x_{1}, x_{2}, x_{3}\right)=(x, y, z) ; P$ is total pressure; $\rho$ is water density; $g$ the gravitational acceleration; $\tau_{i j}$ is turbulent shear stress; and $u_{i}$ is the component of velocity in the specified direction. The transformation

$$
\sigma=(z+h) /(\eta+h)
$$

in which $h(x, y)$ is water depth and $\eta(x, y)$ is free-surface elevation relative to the still-water level, defines the $\sigma$ levels when written in discrete form. Equation (3) 
allows (1) and (2) to be written in conservative form in the $\sigma$ coordinate as

$$
\begin{aligned}
& \frac{\partial \boldsymbol{\Psi}}{\partial t}+\nabla \cdot \boldsymbol{\Theta}(\boldsymbol{\Psi})=\mathbf{S} \quad \text { with } \\
& \nabla=\left(\frac{\partial}{\partial x}, \frac{\partial}{\partial y}, \frac{\partial}{\partial \sigma}\right) .
\end{aligned}
$$

Here, $\boldsymbol{\Psi}$ and $\boldsymbol{\Theta}(\boldsymbol{\Psi})$ are the vectors of conserved variables and the flux vector, respectively, and are functions of $h, \eta, u, v$, and $\omega$, the contravariant component of the vertical velocity. The source term $\mathbf{S}$ includes separate components for bottom slope, pressure gradient, and turbulent mixing. In the numerical model, velocities are evaluated at cell centers, while pressure is calculated along $\sigma$ levels at the top and bottom of each cell. Comprehensive model testing (Derakhti et al. 2015) has demonstrated that NHWAVE can accurately predict depth- and steepness-limited breaking waves using just four to eight $\sigma$ layers; for further details, see Ma et al. (2012) and Derakhti et al. (2015).

Recent adaptations have now enabled NHWAVE to include objects such as ice floes floating on or near its water surface (Orzech et al. 2016a). The effects of a given floe on the fluid are represented using surface boundary conditions in the wave model, mapping the shape of the underside of each floe onto an upper $\sigma$ level slightly below the surface utilizing 3D masks that move with the floe. In open water areas, the $\sigma$ levels continue to track the surface and bed. An immersed boundary method is used along the sides of each floe to determine the lateral effects of the floe on neighboring fluid volumes, determining fluid velocities in partially filled cells by interpolation (Ma et al. 2016). The method produces an additional forcing term, $S_{\mathrm{IB}}$, that is added to the righthand side of (4) for cells along the boundary of the ice floe only. Fluid fluxes and pressure variations are computed from the corresponding accelerations at floe/cell boundaries. The vertical velocity of the fluid along the underside of the floe is determined from the kinematic boundary condition at the floe's bottom boundary elevation $z_{o}$,

$$
\left.w\right|_{z_{o}}=-\frac{\partial z_{o}}{\partial t}-\left.u\right|_{z_{o}} \frac{\partial z_{o}}{\partial x}-\left.v\right|_{z_{o}} \frac{\partial z_{o}}{\partial y} .
$$

In the transformed domain, the contravariant of vertical velocity in the sigma direction must also satisfy $\omega=0$ along both the top and bottom boundaries of the floe.

Extending the implementation of fluid-object interaction that was described in Orzech et al. (2016a), formulations for the drag force have now been incorporated into the coupled model system. In general, the motion of an ice floe follows but does not exactly match that of the surrounding fluid when there are waves. The velocity shear between the floe and the water produces drag forces that act on both the fluid and the ice. Computation of the ice-fluid drag force has been implemented in the wave model, based on the assumption that the velocity profile at the interface follows the distribution of a stably stratified logarithmic boundary layer. Consequently, the drag coefficient $C_{d}$ may be expressed as

$$
C_{d}=\left[\frac{\kappa}{\ln \left(30 \Delta z / K_{s}\right)}\right]^{2},
$$

where $\kappa=0.41$ is the von Kármán constant, $\Delta z$ is vertical grid spacing, and $K_{s}$ is roughness height that can be specified by the user (a value of $1 \mathrm{~mm}$ was used herein). The dynamic boundary conditions at the wave-ice interfaces in the $x-z$ plane, $y-z$ plane, and $x-y$ plane are written as

$$
\begin{aligned}
& \mu_{t} \frac{\partial u}{\partial y}=C_{d}\left|\mathbf{u}-\mathbf{u}_{\mathrm{obs}}\right|\left(u-u_{\mathrm{obs}}\right), \\
& \mu_{t} \frac{\partial w}{\partial y}=C_{d}\left|\mathbf{u}-\mathbf{u}_{\mathrm{obs}}\right|\left(w-w_{\mathrm{obs}}\right) \\
& \mu_{t} \frac{\partial v}{\partial x}=C_{d}\left|\mathbf{u}-\mathbf{u}_{\mathrm{obs}}\right|\left(v-v_{\mathrm{obs}}\right), \\
& \mu_{t} \frac{\partial w}{\partial x}=C_{d}\left|\mathbf{u}-\mathbf{u}_{\mathrm{obs}}\right|\left(w-w_{\mathrm{obs}}\right) \\
& \mu_{t} \frac{\partial u}{\partial z}=C_{d}\left|\mathbf{u}-\mathbf{u}_{\mathrm{obs}}\right|\left(u-u_{\mathrm{obs}}\right), \\
& \mu_{t} \frac{\partial v}{\partial z}=C_{d}\left|\mathbf{u}-\mathbf{u}_{\mathrm{obs}}\right|\left(v-v_{\mathrm{obs}}\right),
\end{aligned}
$$

respectively, where $\mu_{t}$ is the dynamic viscosity; $\mathbf{u}$ and $\mathbf{u}_{\text {obs }}$ represent the fluid velocity and the object velocity, respectively; and $u, v$, and $w$ are components of these velocities in the $x, y$, and $z$ directions, respectively. Equations (7)-(9) are solved numerically in NHWAVE using forward and backward differences and result in modified fluid fluxes along the edges of the floating objects. The results of these computations are also used to compute drag forces acting on the ice floes, which are then passed to LIGGGHTS.

\section{b. Ice model}

To simulate the physics of ice floes at submeter scales, the coupled system employs the open-source DEM software package LIGGGHTS. The software is a modified version of the molecular dynamics simulator LAMMPS (Plimpton 1995) that is designed to be more suitable for simulations involving larger-scale particles 
and objects. In the present configuration, ice floes are built from smaller elements by defining bonding forces that act within a random close packing of nearly identical spheres.

The spherical discretization of ice elements is used initially to establish relative positions and internal bond angles. After these have been determined, a Voronoi tesselation technique is employed to delineate element shapes and to compute volume, mass, and density. Interelement forces are assumed to be elastic, with a linear constant of proportionality. Critical fracture stresses are specified in both normal and tangential directions so that ice floes may both deform and fracture in response to wave-generated stresses. The ice floe equation of motion includes internal contact and bond forces as well as external forces from gravity, buoyancy, fluid pressure gradients, and drag. Bond normal stress $\sigma_{b}$ and shear stress $\tau_{b}$ are expressed as follows:

$$
\begin{gathered}
\sigma_{b}=k_{b, n} \delta_{n}+0.5 D k_{b, s} \theta_{s}<\sigma_{b, \text { crit }}, \\
\tau_{b}=k_{b, s} \delta_{s}+0.5 D k_{b, n} \theta_{n}<\tau_{b, \text { crit }},
\end{gathered}
$$

where $\delta$ represents the relative displacement of two elements in the normal $(n)$ and tangential $(s)$ direction, $D$ is the element diameter, and $\theta$ is the bond deformation angle. The normal and tangential bonding coefficients $k_{b}$ are computed from

$$
\begin{aligned}
& k_{b, n}=E / D, \\
& k_{b, s}=k_{b, n} /[2(1+\nu)],
\end{aligned}
$$

where $E$ is Young's modulus for ice and $\nu$ is Poisson's ratio. When either $\sigma_{b}$ or $\tau_{b}$ exceeds the critical value [ $\sigma_{b, \text { crit }}$ or $\tau_{b, \text { crit }}$ in (10) and (11), respectively], the bond between two elements will break.

\section{c. Model coupling}

The software for coupling the (Fortran based) NHWAVE and (C ++ based) LIGGGHTS models was developed within the LIGGGHTS framework. The primary subroutines were written in $\mathrm{C}++$, and each was paired with a corresponding Fortran header to facilitate access by NHWAVE. To jointly compile the separate wave and ice models, the complete LIGGGHTS code, including the coupling subroutines, is first compiled in $\mathrm{C}++$ as a shareable library. The library, along with the coupling header files, is incorporated into the Fortran compilation of NHWAVE. The final executable program can then access all necessary DEM utilities and call on LIGGGHTS to perform ice-related computations as needed.
Information that is passed from waves to ice includes the fluid forces on the ice and the surface elevation in each cell. At the first time step, the wave model also passes information on the number and dimensions of the fixed fluid cells. Fluid-based forces acting on the ice, including nonhydrostatic pressure and drag, are calculated by the wave model. Buoyancy is calculated in the ice model, where it can be determined more accurately. As illustrated in Fig. 1, LIGGGHTS "sees" the ice in its true form, while NHWAVE represents the ice as a conglomeration of approximate boxes whose vertical extent is defined by the $z_{b}$ and $z_{t}$ values obtained from the ice model. In determining buoyancy, LIGGGHTS utilizes the surface elevation in each cell to compute the exact position of each element relative to the water line and identify which elements are subject to the force. It then adds these force vectors to the interelement forces and those received from the wave model to get the net forcing on each element.

Information passed from ice to waves includes elevations of the highest $\left(z_{t}\right)$ and lowest $\left(z_{b}\right)$ ice elements, average velocities, average accelerations, and total volume of the ice elements in each fluid cell (vol). The wave model does not "see" the individual ice elements; instead it sees the continuous ice as either fully or partially occupying a given cell. An ice block is considered to occupy all contiguous fluid cells for which vol is greater than half of the total cell volume between $z_{b}$ and $z_{t}$. At each wave time step a $\sigma$ layer just below the surface is mapped to the exact elevation of the underside of the ice floe (i.e., to $z_{b}$ ). In the case of overtopping (not currently enabled), this $\sigma$ layer is instead mapped to the elevation of the top of the floe (i.e., to $z_{t}$ ) and the next deeper $\sigma$ layer is mapped to the underside of the floe.

In typical simulations, the wave model time step is dynamically assigned in the range $10^{-4}-10^{0} \mathrm{~s}$, while the DEM time step is $10^{-5} \mathrm{~s}$. The coupling time step is presently the same as the wave model time step.

\section{System evaluation}

Preceding the creation of the coupled system, the stand-alone wave and ice models were independently configured and validated for processes and properties specific to their separate domains (Orzech et al. 2016a; Bateman et al. 2016); a brief summary is provided below in section 3a. More recent evaluations of the coupled system, including single and multiple ice blocks in different wave conditions as well as a comparison case with pancake ice data, are detailed in sections $3 \mathrm{~b}$ and $3 \mathrm{c}$. In all of the tests below, ice density was set to $920 \mathrm{~kg} \mathrm{~m}^{-3}$ and fluid density was fixed at $1025 \mathrm{~kg} \mathrm{~m}^{-3}$. 

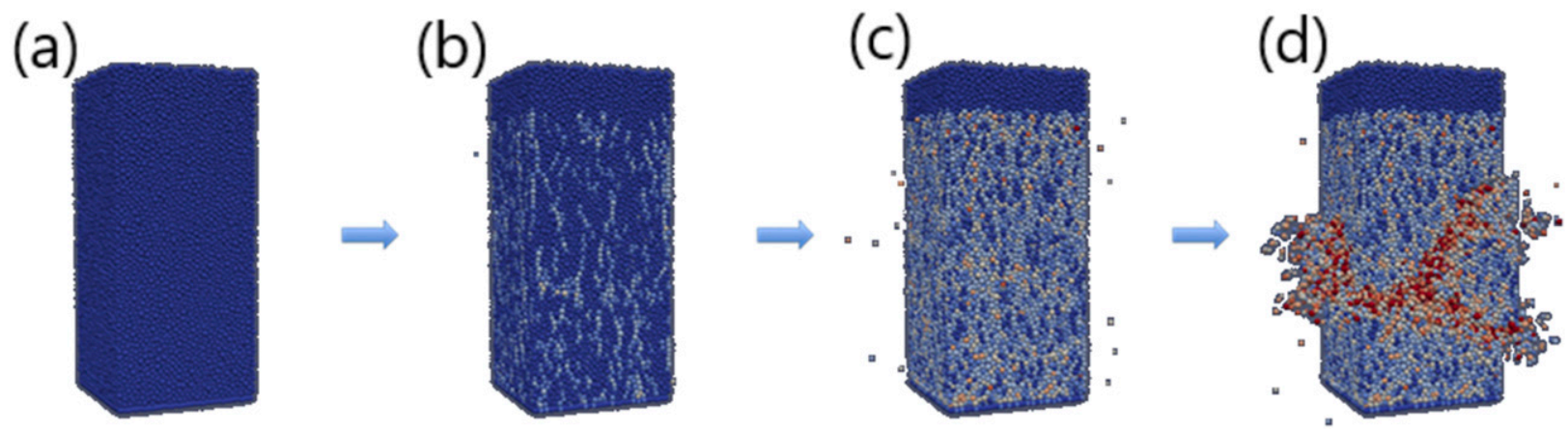

FIG. 2. Snapshots from the ice compression test (Bateman et al. 2014), showing the condition of bonds at times (a) 0.0, (b) 1.0, (c) 1.8, and (d) $2.0 \mathrm{~s}$. A $2.5 \mathrm{~m} \times 2.5 \mathrm{~m} \times 5 \mathrm{~m}$ block was subjected to uniform compressive force, with failure [(d)] occurring at a compressive stress of approximately 3.4 MPa. Color of particles indicates the number of bonds broken, from 0 (blue) to 6 (red).

\section{a. Stand-alone validation and calibration}

The stand-alone wave model validations focus on the reflection, transmission, refraction, and generation of ocean surface waves by objects that are fixed or in controlled motion at the surface (Orzech et al. 2016a). The objects are artificial and rigid and do not move in response to wave forcing. Model output is compared with analytical solutions for fixed 2D blocks and with wave generation by a vertically oscillating sphere in a laboratory experiment. For model-theory comparisons of wave energy transmitted through a group of blocks $\left(K_{t}\right)$ and wave energy radiated by heaving, swaying, and rolling blocks, normalized RMS errors are generally on the order of $1 \%-2 \%$. For the laboratory case, errors in modeled versus measured wave height are also on the order of $1 \%-2 \%$. A comparative simulation with steeper waves qualitatively demonstrates the efficiency of the wave model relative to a volume of fluid (VOF) model, and a second qualitative simulation using a larger-scale domain produces reasonable results for wave behavior in a realistically distributed field of ice floes. For additional details, see Orzech et al. (2016a).

The stand-alone ice model validations (Bateman et al. 2016) test and calibrate the material behavior of the DEM ice blocks by subjecting them to a series of virtual stress tests (e.g., Fig. 2). Stress and strain responses of ice blocks are tracked for flexural, compressional, and tensile tests, and critical fracture stresses are identified for a range of bonding strengths. Critical bonding stress values for ice elements are tuned so that the macroscopic fracturing behavior of virtual ice blocks fits within ranges measured for tests with actual sea ice (e.g., $S_{\text {flex }}=0.1-1.0 \mathrm{MPa}$; $S_{\text {comp }}=0.8-1.2 \mathrm{MPa} ; S_{\text {tens }}=0.2-0.8 \mathrm{MPa}$; Timco and Weeks 2010). For microscale critical bond strengths ranging from 0.1 to $2.0 \mathrm{MPa}$ (assuming $\sigma_{b, \text { crit }}=\tau_{b, \text { crit }}$ ), the corresponding macroscale flexure, tension, and compression fracture limits are found to be roughly $15 \%-40 \%$ lower than the microscale values, depending on the type of test (Fig. 3). Comparing the results to published laboratory measurements indicates that virtual ice behavior best approximates that of real sea ice for critical bond stress values between 0.75 and $1.25 \mathrm{MPa}$; for additional details, see Bateman et al. (2018, manuscript submitted to Mech. Res. Commun.).

\section{b. Preliminary coupled tests}

An initial evaluation of the coupled system was performed by running a still-water test with a vertically "bouncing" block and tracking the damped motion of the center of mass. This was followed by an examination of the fluid and ice properties and behavior for a single ice floe in a monochromatic wave field.

\section{1) BOUNCING BLOCK}

In this simulation an ice block 1-m thick and $10 \mathrm{~m} \times$ $10 \mathrm{~m}$ in area was positioned roughly $20 \mathrm{~cm}$ above neutral buoyancy at the water surface in the center of a tank with an area of $100 \mathrm{~m} \times 100 \mathrm{~m} \times 5 \mathrm{~m}$, and then released and allowed to oscillate. The resulting motion of the block's center of mass (Fig. 4) was steadily damped over time as the block position approached a neutral state. An idealized damped harmonic oscillation was computed utilizing the mean period and amplitude decay rate of the block and is included in the figure for comparison. As the figure illustrates, the block's oscillation period was somewhat lengthened during the early cycles ( $T=2.4 \mathrm{~s}$ ) then gradually stabilized to $T \approx 2.2 \mathrm{~s}$ as the amplitude decreased. This is consistent with the experimental results (e.g., Chung 2008), which indicate that the period of an oscillating object at the water surface is greater for larger displacements. For a $100-\mathrm{m}^{3}$ ice block with a mass of approximately $9.2 \times 10^{4} \mathrm{~kg}$, the natural harmonic frequency is about $0.5 \mathrm{~Hz}$, which is roughly consistent with the period found here. The reduction 


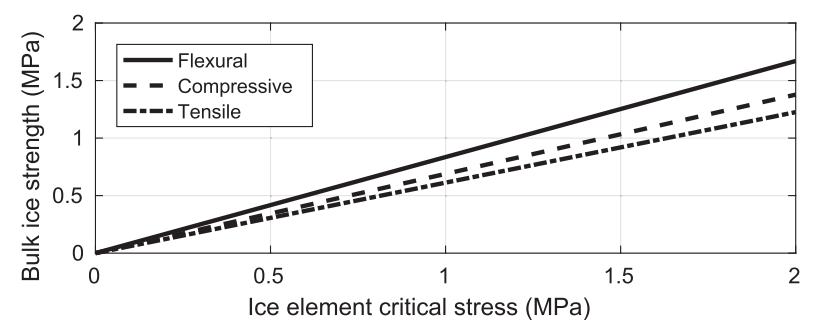

FIG. 3. Linear best-fit results for macroscale flexural, compressive, and tensile strength of ice blocks as a function of microscale ice element critical bond stress value (based on Bateman et al. 2016). For these results, it is assumed that normal and tangential bond stress values are equal (i.e., $\sigma_{b}=\tau_{b}$ ).

in amplitude is fairly regular, with a damping factor $\left(K=A_{n+1} / A_{n}\right)$ ranging from 0.82 to 0.92 over the oscillation periods shown.

Upon examining the floe's orientation at each time step, we observed that the floe did gradually begin to pitch and roll somewhat during its oscillations, primarily in the $y-z$ plane. By the time step of the thirteenth peak in Fig. 4, (i.e., near $t=26 \mathrm{~s}$ ), one end of the block was approximately $1.5 \mathrm{~cm}$ higher than the other end in the $y-z$ plane. With further investigation, we determined that the pitching and rolling were caused by slight irregularities in the distribution of elements within the block, which had slightly fewer elements on one side than on the other. The imbalance produced a different buoyancy forcing on each side, leading to variations in the frequency of oscillation. While it would be possible to preconfigure a perfectly symmetric element distribution, a mildly skewed configuration is more realistic for ice floes in general. Despite the irregularities, the overall bouncing motion of the floe continued to damp and approach a stable state. The results confirmed that although the coupled model can be slightly disrupted by more extreme ice displacements, it recovers quickly and handles smaller vertical oscillations reasonably well.

\section{2) Single Floe AND UNiform WAVES}

In this monochromatic wave test, a neutrally floating block was allowed to move freely in response to $0.5 \mathrm{~m}$ waves of a single frequency $(0.2 \mathrm{~Hz})$. Waves were generated at $x=100 \mathrm{~m}$ in a $400 \mathrm{~m} \times 200 \mathrm{~m}$ wave flume, with a $10 \mathrm{~m} \times 10 \mathrm{~m} \times 1 \mathrm{~m}$ ice block centered at $x=$ $205 \mathrm{~m}, y=100 \mathrm{~m}$, and neutral buoyancy. Model output, including fluid pressure, velocities, and free-surface elevation along with ice floe position, were recorded at synchronized regular intervals. To better illustrate the pressure gradient forces, nonhydrostatic pressure output from NHWAVE was converted to dynamic pressure by adding the contribution of the free surface:

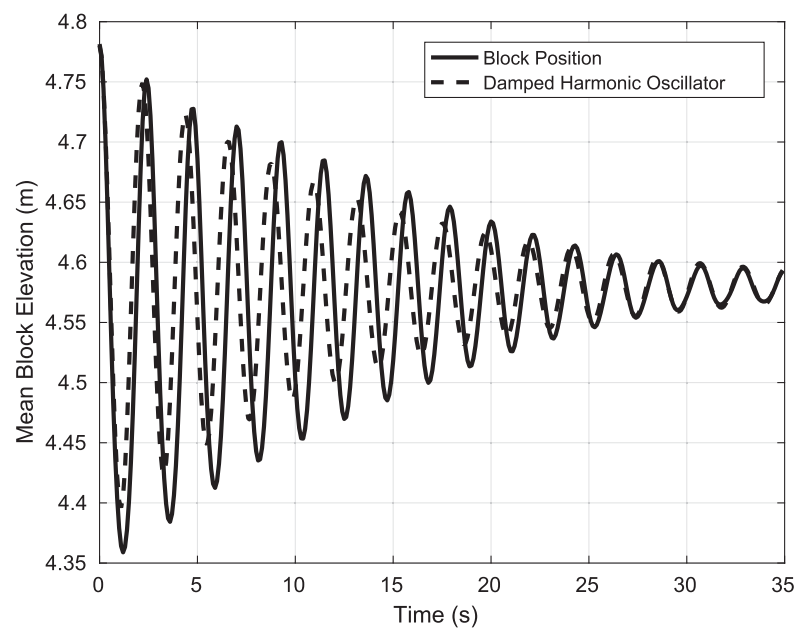

FIG. 4. Position of center of mass vs time for a $10 \mathrm{~m} \times 10 \mathrm{~m} \times 1 \mathrm{~m}$ ice floe, starting from an initial point roughly $20 \mathrm{~cm}$ above the stillwater level (solid line). Damped harmonic oscillation; fitted based on mean block oscillation period and peak elevation decay rate (dashed line).

$$
P_{\mathrm{dyn}}=P_{\mathrm{NH}}+\rho g \eta .
$$

Dynamic pressure was more variable underneath the floe than at other locations along the flume (Fig. 5). The variations in $P_{\text {dyn }}$ were a consequence of the mildly oscillating floe's effects on fluid fluxes near its boundaries, where it acted as a barrier to normally directed fluxes and created shear drag on tangential fluid flow, as in (7)-(9). At $t=43 \mathrm{~s}$ (upper panel), the upward-directed fluid flux (mean vertical velocity $\approx 0.2 \mathrm{~m} \mathrm{~s}^{-1}$ ) is accelerated as it encounters the underside of the more rapidly upward-moving ice floe (mean vertical velocity $\approx 0.25 \mathrm{~m} \mathrm{~s}^{-1}$ ), causing dynamic pressure to decrease (from 1.5 to $0.8 \mathrm{kPa}$, as indicated by the orange/green contours). Pressure increases at the leading (left) edge of the floe, as rightward-directed crest velocities encounter the floe edge and decelerate (bright yellow contour). At $t=46 \mathrm{~s}$ (lower panel), leftward-directed fluid flux under the floe is intensified, as it is partially blocked by the dipping ice edge. This produces a corresponding reduction in dynamic pressure (from -1.0 to $-2.0 \mathrm{kPa}$ ) in areas of accelerated flow (darker blue contours).

An examination of time series of the free surface and the floe's average vertical position indicates that the motion of the floe was governed by the wave state and slightly lagged the motion of the regular waves (Fig. 6). The lag was approximately $1 \mathrm{~s}$, or roughly $20 \%$ of the wave period. The amplitude of the mean floe motion was about half of the amplitude of the waves (note that wave amplitude initially increased but then stabilized as the first waves propagated away from the wavemaker and 

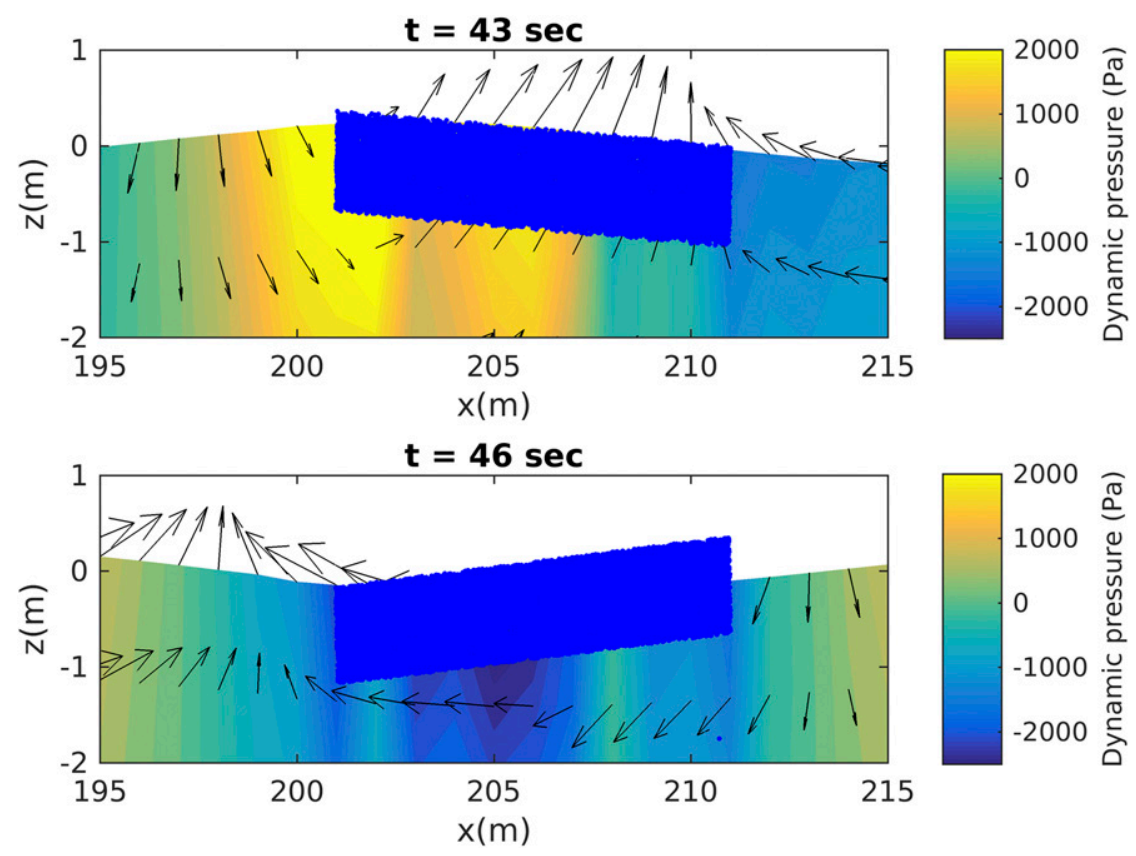

FIG. 5. Single floe in monochromatic waves $(x-z$ transect along $y=100 \mathrm{~m}$, middle of wave flume). Color shading indicates dynamic pressure values, following the color bar on the right side. Arrows depict velocity, and blue box is ice floe. Floe shape appears slightly distorted as a result of axis scaling. (Note: Velocity vectors for fluid cells containing the ice floe are set to mean floe velocities for the respective cells.)

the simulation approached a steady state). The effect of the floe on the surrounding waves is visible in plan view as a mild wake in wave crests leeward of the ice floe (Fig. 7, top panel). When the background waves themselves are removed, the remaining surface perturbation caused by the floe is revealed to be a concentric wave pattern radiating outward from the floe's location (Fig. 7, bottom panel). The perturbation pattern is produced by the out-of-phase motion of the floe relative to the water surface; it has a wavelength similar to that of the background waves $(\approx 35 \mathrm{~m})$.

\section{c. Multifloe evaluations}

To increase the complexity of the simulations and to allow for some comparison of coupled model output to measured data, two sets of model tests were conducted that included multiple ice floes of varying size. The first set of tests focused on the reflection and transmission of waves by ice floe fields of several different size distributions [section $3 \mathrm{c}(1)]$. The second set created hindcasts based on measurements of waves in pancake ice that were obtained during a 2015 Arctic expedition [section 3c(2)].

\section{1) REFLECTION AND TRANSMISSION}

Montiel et al. (2016) use a model based on linear potential flow and elastic plate theory to study the reflection and attenuation of ocean waves in fields of circular ice floes with diameters obeying a power-law distribution, randomly positioned in rectangular regions they call "slabs." To investigate the coupled system's estimates of wave reflection and transmission by multiple ice floes, we designed a set of experiments to roughly recreate selected cases from Montiel et al. (2016) in a wave flume. The floe field composition was determined by following the prescription for slabs of assorted floes that is provided in their appendix B. In the present simulations, the initial full slab measured $200 \mathrm{~m} \times$ $1.6 \mathrm{~km}$ and included 10 randomly distributed floe types.

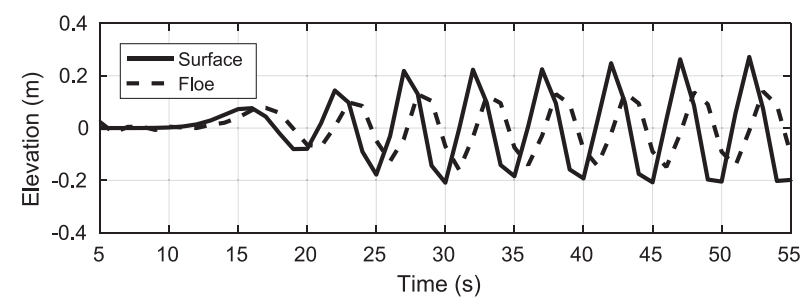

FIG. 6. Time series of free-surface elevation (solid line) and average vertical floe position (dashed line). Surface elevation is measured at $x=205 \mathrm{~m}$, corresponding to the center of floe. Floe oscillations consistently lag those of the free surface by approximately $1 \mathrm{~s}$. 

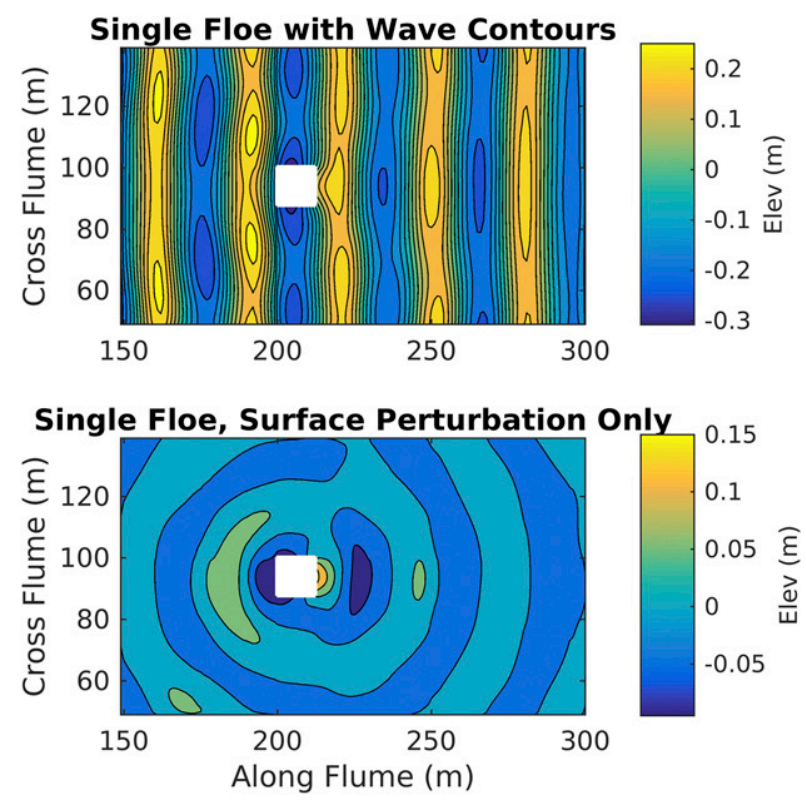

FIG. 7. Single floe (white box) in monochromatic waves at $t=$ $46 \mathrm{~s}$, plan view with wave contours. (top) Full wave contours; ice floe interaction is visible as a wedge-shaped wake in waves leeward (right) of the floe. (bottom) Contours of surface perturbation only; primary background wave signal has been subtracted to show net effect of floe on the surface.

Floe sizes ranged from $a_{\min }=10 \min$ to $a_{\max }=91 \mathrm{~m}$, matching those in Montiel et al. (2016) except that the floes used here were square in shape rather than circular (to avoid ice fragility and excessive fracturing that occurred with circular floes in LIGGGHTS). In limited testing, averaged reflection and transmission rates for square and circular floe types were found to be within $5 \%$.

Three floe configurations were chosen for the tests to follow, featuring just the largest sizes in order to keep computation times achievable. The configurations included slabs retaining the four largest floe types (i.e., 64, 73,82 , and $91 \mathrm{~m}$ ), the two largest floe types (i.e., 82 and $91 \mathrm{~m}$ ), and a single floe type ( $91 \mathrm{~m}$ only). As in the cited study, each sparser slab was generated from the original full slab by removing all floes with a side length smaller than the included types. Each partial slab was positioned along the center line of a $4 \mathrm{~km} \times 2 \mathrm{~km}$ wave flume, extending from $x=2 \mathrm{~km}$ to $x=2.2 \mathrm{~km}$ and covering almost the entire width of the flume (Fig. 8). Unidirectional waves of period $T$ equal to 6 and $9 \mathrm{~s}$ were generated roughly $1 \mathrm{~km}$ in front of each ice floe configuration and propagated into and through the floes. Numerical sponges were employed along flume boundaries to absorb outward-radiating wave energy.

Each of the six simulations was allowed to run for sufficient time to enable the waves to reach the far end of

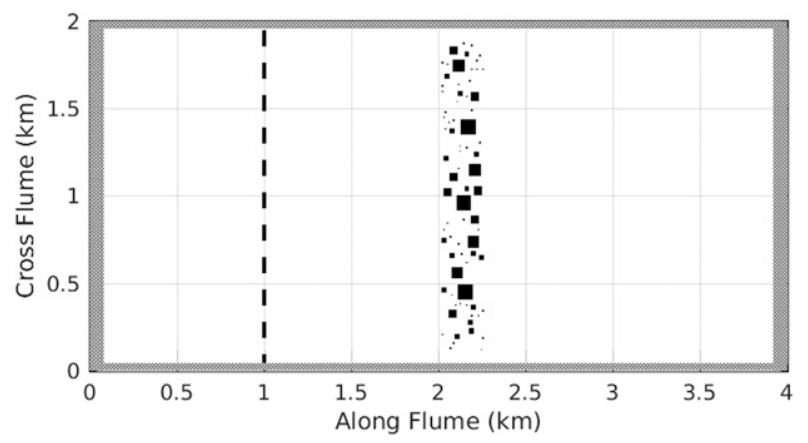

FIG. 8. Plan view of sample ice floe slab in wave flume. Dashed line at $x=1 \mathrm{~km}$ indicates wavemaker location, and boundary sponges are depicted with cross-hatching.

the flume, after which the wave energy levels were measured. The mean wave energy per unit surface area $(E)$ was estimated using spatial averages of representative kinetic (KE) and potential (PE) energy for selected regions in front of and behind the floe fields,

$$
\begin{aligned}
E & =\mathrm{KE}+\mathrm{PE}, \\
& =\frac{1}{L_{x} L_{y}}\left[\sum_{A \cdot h} \frac{\rho}{2}\left(u^{2}+v^{2}+w^{2}\right) \Delta x \Delta y \Delta z+\sum_{A} \frac{\rho g}{2} \eta^{2} \Delta x \Delta z\right],
\end{aligned}
$$

where $L_{x}$ and $L_{y}$ are the horizontal dimensions of each region. Incident plus reflected energy, $E_{i}+E_{r}$, was computed for a region immediately in front of the ice floes (i.e., $x=1.75-2 \mathrm{~km}, y=0.5-1.5 \mathrm{~km}$ in Fig. 8). Mean incident energy $E_{i}$ was computed for this region from a separate simulation featuring the same waves but with floes removed. Average reflected energy $E_{r}$ was extracted by taking the difference of these two results. Mean transmitted energy $E_{t}$ was computed for a region immediately behind the ice floes in the flume $(x=2.3-2.55 \mathrm{~km}, y=0.5-1.5 \mathrm{~km})$. Wave reflection and transmission coefficients were calculated as $K_{r}=E_{r} / E_{i}$ and $K_{t}=E_{t} / E_{i}$, respectively. An unobstructed transmitted energy, $E_{t 0}$, was also computed for this region from the simulation without floes. A small amount of amplitude reduction was observed in waves propagating in the ice-free flume $(\sim 5 \%$ over the length of the domain; likely resulting from numerical dissipation).

Reflection and transmission results are summarized in Table 1. The wave period is listed in the first column, and the second column describes the configuration (i.e., the number of different floe types), while the third column lists the total number of ice floes actually contained in the reduced slab. As anticipated, wave reflection $\left(K_{r}\right.$, column 6) was consistently larger for 6-s waves than for 
TABLE 1. Energy reflection and transmission by floe slabs; $T=6$ and $9 \mathrm{~s}$.

\begin{tabular}{cccccccr}
\hline \hline$T(\mathrm{~s})$ & $\begin{array}{c}\text { Configuration } \\
\text { (type) }\end{array}$ & No. of floes & $E_{i}$ & $E_{r}$ & $K_{r}$ & $K_{r, \text { mon }}$ & $E_{t}$ \\
\hline 6 & 4 & 7 & 1.85 & 0.59 & 0.32 & 0.42 & 1.26 \\
6 & 2 & 3 & 1.85 & 0.53 & 0.29 & 0.28 & 1.32 \\
6 & 1 & 2 & 1.85 & 0.39 & 0.21 & 0.14 & 1.46 \\
9 & 4 & 7 & 7.79 & 1.43 & 0.18 & 0.10 & 5.82 \\
9 & 2 & 3 & 7.79 & 1.02 & 0.13 & 0.05 & 6.60 \\
9 & 1 & 2 & 7.79 & 0.63 & 0.08 & 0.04 & 6.69 \\
\hline
\end{tabular}

9-s waves, and values for both wave periods decreased as floes were removed from the slab.

Following the same pattern, results from Montiel et al. (2016) also indicate that wave reflection is strongly influenced by the floe types that are included in the slabs. For a given frequency, they report that wave reflection remains nearly constant as long as the floe distribution includes both smaller and larger floe types, but it declines fairly rapidly if the minimum allowed floe diameter is increased and only a few large floes are left in the slab. For similar $[4,2,1]$ floe-type configurations to those used here, Montiel et al. (2016) obtain values for $K_{r, \text { mon }}$ of approximately $[0.42,0.28,0.14]$ for 6 -s waves and $[0.10,0.05,0.04]$ for 9-s waves (see Table 1 , column 7; and Fig. 4 in Montiel et al. 2016). The present results have somewhat different spacing but a similar mean value for 6-s waves, while our estimated $K_{r}$ for 9-s waves is slightly higher in each case.

Our estimates of transmitted wave energy $\left(K_{t}\right)$ were roughly complementary to reflection values (Table 1 , column 9). As expected, transmission was generally greater for 9-s waves than for 6-s waves, and transmission increased as floes were removed from the slab. Transmission estimates for these cases were not made available in Montiel et al. (2016). Summing $K_{r}$ and $K_{t}$ in the six cases results in values ranging from 0.80 to 0.98 , with the lower values all occurring for the 6 -s waves. This suggests that the 6-s waves were more greatly dissipated by interactions with ice floes than were the 9-s waves.

\section{2) HiNDCASTS OF PANCAKE ICE FIELD DATA}

Recent field campaigns have yielded several excellent datasets incorporating simultaneous measurements of ice and ocean (e.g., Thomson 2015; Kohout et al. 2014; Thomson et al. 2013). Here we use data from one major Arctic experiment to evaluate wave attenuation predictions made by the coupled wave-ice model. In October-November 2015, scientists participating in the ONR Arctic Sea State Department Research Initiative (DRI) cruise recorded several comprehensive, synchronous datasets of wave propagation in a growing MIZ (Thomson et al. 2013). Although the cruise dealt primarily with smaller, thinner "pancake" ice, these datasets included local MIZ ice distribution, size, and thickness information together with spectral wave data from both MIZ and open water locations, as well as regional current velocities. For one significant wave event (11-14 October), an analysis was performed of wave attenuation by the thin, variable ice cover (Rogers et al. 2016). Over the 4-day period, the research team compiled extensive measurements of ice configuration and wave time series along a roughly $137-\mathrm{km}$ transect. The follow-up study used an inversion method with buoy-measured wave spectra, in combination with the WAVEWATCH III model (Tolman 1997), to investigate the dissipation of wave energy by different types of primarily pancake and frazil ice.

To create a scenario resembling the field-based conditions of Rogers et al. (2016), the uncoupled LIGGGHTS software was employed to build artificial circular and elliptical ice pancakes of dimensions similar to those in the field dataset [i.e., $O(1-2) \mathrm{m}$ horizontal, $O(20) \mathrm{cm}$ vertical]. Interelement bonding strength was set near the lower end of the range determined in the virtual ice stress tests described in section 3a. These pancakes were then replicated and placed into a narrow $500 \mathrm{~m} \times 60 \mathrm{~m}$ area with a 30-m-deep flume (Fig. 9). The pancakes initially were closely spaced and covered a rectangular area roughly $100 \mathrm{~m} \times 20 \mathrm{~m}$, centered at $(x=250 \mathrm{~m}$,

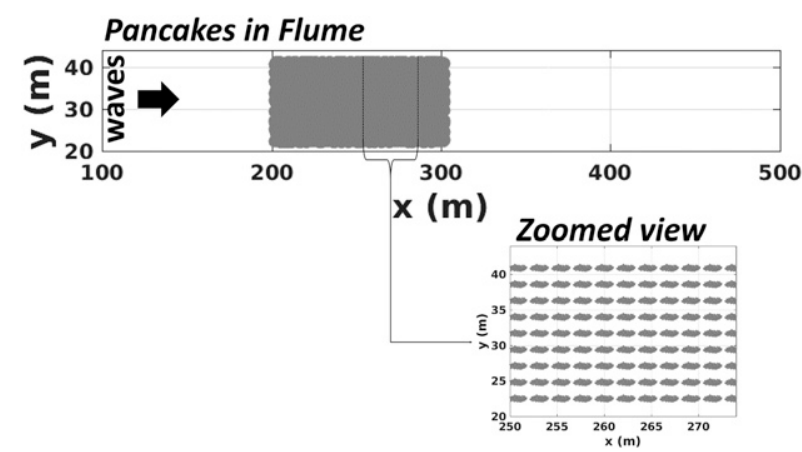

FIG. 9. Model setup for pancake simulations, with portion enlarged to show individual pancake floes. 
$y=30 \mathrm{~m}$ ). Pancakes were not allowed to create new bonds, but existing bonds could be broken if stresses were large. Monochromatic waves of several different frequencies were generated by a wavemaker located at $x=100 \mathrm{~m}$, and incident and transmitted wave energy were estimated for each wave type using (15). Wave attenuation was calculated in each case for comparison to results from Rogers et al. (2016).

A simplified representative wave height $H$ was estimated using total fluid energy $E$,

$$
H \sim \sqrt{E} .
$$

We assume here, as in the earlier study, that the attenuation of wave height in pancake ice can be expressed as

$$
H(x)=H_{o} e^{-k_{i} x},
$$

where $k_{i}$ is the exponential decay rate of amplitude in space, defined for this study as

$$
k_{i}=0.01 \ln \left[\frac{H_{o}}{H(100)}\right] \text {. }
$$

Time and spatial averages in the regions $x=120-150 \mathrm{~m}$ and $x=350-380 \mathrm{~m}$ were used to determine $H_{o}$ and $H(100)$, respectively.

Monochromatic waves with frequency values 0.05 , $0.075,0.10$, and $0.15 \mathrm{~Hz}$ were propagated through the $100-\mathrm{m}$ field of ice pancakes described above in four separate simulations. Two sets of tests were run: a first set in which each wave train had a constant amplitude of $0.5 \mathrm{~m}$ and a second set in which amplitudes were varied to maintain a constant steepness for all wave trains. Each test case was also rerun without ice to quantify purely numerical dissipation, which was then added back to transmitted energy levels in the original test cases. For this frequency range, Rogers et al. (2016) found that the average value of $k_{i}$ with a pancake/ frazil ice mixture rose fairly steadily from $10^{-6}$ to $10^{-5} \mathrm{rad} \mathrm{m}^{-1}( \pm 50 \%)$.

The results from the two sets of tests are compared to those from Rogers et al. (2016) in Fig. 10. In the constant amplitude tests (blue asterisks), the dissipation rate parameter $k_{i}$ consistently increases with increasing frequency, in a manner similar to that measured by Rogers et al. (2016) (red dashed line). However, here the coupled model suggests that dissipation will be greater by an order of magnitude than the values obtained for the same frequency range under pancake/frazil ice conditions in that analysis. In the constant steepness tests (black circles), $k_{i}$ also climbs after remaining nearly constant at lower frequencies. It is somewhat closer in magnitude to the attenuation levels of Rogers et al.

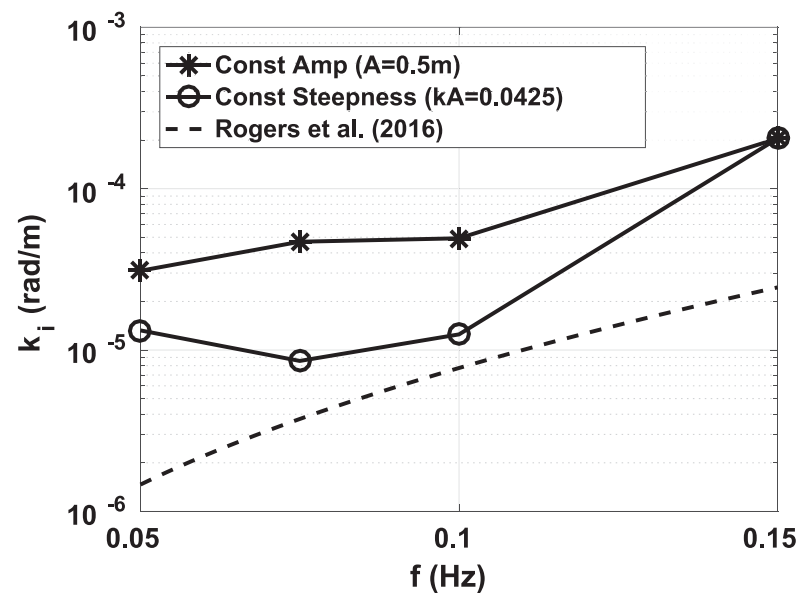

FIG. 10. Model results for $k_{i}$ vs frequency from multiple simulations with constant wave amplitude (asterisks) or constant wave steepness (circles). Best fit to data from Rogers et al. (2016) (dashed line).

(2016), particularly at typical wind and swell wave frequencies of $0.075-0.1 \mathrm{~Hz}$. Standard deviations of our estimated values were also fairly high $[O(50) \%]$, overlapping with those from the field study.

Limitations stemming from relative model scaling requirements in NHWAVE made it computationally too expensive to run simulations for waves with frequency higher than $0.15 \mathrm{~Hz}$. See section 4 for further discussion of this issue and the abovementioned results.

\section{Discussion and conclusions}

A system for modeling small-scale wave-ice interactions in the MIZ has been developed by coupling the nonhydrostatic fluid model NHWAVE to the discrete element model LIGGGHTS. The system tracks energy and momentum in both fluid and ice domains, simulating wave reflection, diffraction, and transmission as well as ice floe flexure, fracturing, and collisions. The coupled system was first evaluated with two basic simulations featuring a single ice block, first in still water and then in monochromatic waves. Subsequently, the system was applied to two more complex scenarios, the first involving multiple ice floes with varying size distributions and the second a comparative simulation based on results from a 2015 field experiment featuring pancake ice in the Beaufort Sea.

In the initial single-block tests [section $3 b(1)$ ], the wave-ice model performed generally as expected, although fully quantitative validations were not yet possible. For an ice floe that was released from a point above neutral buoyancy, the predicted oscillatory motion was very close to periodic and was damped steadily 
toward a stable, neutral position. Results were compared to a time series for an idealized damped harmonic oscillator based on the block's amplitude envelope and mean period. The initial oscillation period of the ice block was slightly longer than the mean, and initial amplitudes were slightly larger, but these variations disappeared as the oscillation amplitude decreased.

While experimental validation is not yet available, a simplified calculation may be performed to roughly evaluate the rate of energy loss suggested by the coupled model for the bouncing block. As the first two peaks of the block's oscillation differ by $3 \mathrm{~cm}$, potential energy lost between the first and second peaks can be estimated as the energy change caused by shifting the block $3 \mathrm{~cm}$ downward. For ice of density $920 \mathrm{~kg} \mathrm{~m}^{-3}$, this results in a loss of about $27 \mathrm{~kJ}$. The block's lost energy must be either dissipated by drag effects or absorbed by increased motion and displacement of the water. Using a drag coefficient $C_{d}=0.5$ (based on a Reynolds number of $4 \times 10^{6}$ ), the quadratic formulation for drag is integrated over the first full block oscillation. With velocity estimated as the change of block position per 0.1-s time step, the computation produces an estimated drag of $3.6 \mathrm{~kJ}$. By summing up the potential energy of all surface displacements and the kinetic energy of all fluid motion throughout the domain at the end of the first bounce, the increase in total energy of the water is calculated to be approximately $23 \mathrm{~kJ}$. Comparing the combined drag loss and fluid energy change $(26.6 \mathrm{~kJ})$ to the block's potential energy loss $(27 \mathrm{~kJ})$, the model result for the large first oscillation appears reasonably consistent. The abovementioned computation of drag is of course subject to some uncertainty, and the model itself may have underestimated drag losses, as vortex shedding is not included in the coupled system. Nevertheless, the two total energy values differ by less than $2 \%$. Error magnitudes will likely decrease further with more closely spaced $\sigma$ levels and/or smaller oscillation amplitudes that are more typical of field conditions.

Upon closer examination of the floe itself, we found that the ice block gradually developed a slight pitching and rolling as it continued to oscillate. This wobbling likely resulted from irregularities that were inherent in the floe itself (i.e., slightly more mass on one side than another), which were a consequence of the randomized bonding process used in the original construction of the floe with LIGGGHTS. These irregularities will generally be reduced as floe size and thickness are increased (relative to individual element diameters).

It should be noted that virtual ice floes in this system are designed to approximate the average behavior of real ice floes. While they have some variability as seen above, they do not include microscale features such as variable salinity, random irregularities, partial fractures, or weak areas caused by ponding. Although such variations would be configurable, modeling them in individual floes would contribute little to the overall goal of validating large-scale attenuation parameterizations while drastically increasing the computational requirements of each simulation. The ice in the floes has been shown to move, bend, and fracture in a manner typical of measured results for sea ice. The wave interactions of a group of such floes should thus be reasonably representative of the interactions of a similar group of randomly varying real ice floes in the field, providing important insights into the relative importance of each small-scale process in accelerating wave attenuation and ice edge retreat.

In simulations with a single block in monochromatic waves [section $3 b(2)$ ], the modeled dynamic fluid pressure underneath the floating block was directly affected by interactions between the waves and the block. Fluid velocities were altered along the lower edge of the ice floe, with upward-directed velocity vectors slowing as they encountered the solid surface above them and leftward-directed vectors accelerating to maintain continuity as they were redirected to a longer path beneath the dipping block edge. The corresponding changes in pressure qualitatively fit with expectations based on the Bernoulli effect, in a manner similar to airflow over an airplane wing.

For a series of tests incorporating distributions of variably sized ice floes [section 3c(1)], calculated estimates of reflection were similar to results from Montiel et al. (2016), though they varied somewhat differently for each configuration. In agreement with results from the earlier study, reflection was consistently greater for floe distributions containing greater numbers of floes and floes of smaller size. Estimates of wave transmission were generally reasonable, based on the corresponding reflection values, and the overall energy was more attenuated for 6 -s waves $\left(K_{r}+K_{t} \approx 0.8\right)$ than for 9-s waves $\left(K_{r}+K_{t} \approx 0.9\right)$. Numerical dissipation appeared to be minimal over the relatively short distance through the floe fields. However, such dissipation can pose a problem for the coupled system when it becomes necessary for waves to pass through broader regions of ice floes.

In the final set of comparative simulations [section $3 c(2)]$, the coupled wave-ice system correctly captured the generally increasing trend of wave attenuation with frequency in pancake ice. Modeled attenuation estimates were based solely on waves passing through idealized, uniform pancake ice and overestimated the dissipation rate parameter $k_{i}$ in comparison to results from Rogers et al. (2016). Numerical dissipation effects may have played a role in overdamping waves for these 
cases. However, photo images of ice pancakes using Surface Wave Instrument Float with Tracking (SWIFT) for the measurement period (Rogers et al. 2016, their Fig. 3) also suggest that the pancake distribution in the field may have been significantly sparser than the relatively tight spacing that was assumed in this hindcast (Fig. 9), with much of the remaining space occupied by open water or frazil ice. If so, this mismatch may be the primary reason for the coupled system's overestimate of attenuation.

Earlier results from the Weddell Sea (Doble et al. 2015) suggest, in contrast to Rogers et al. (2016), that the value of $k_{i}$ for a similar pancake distribution may in fact be significantly higher. Depending on pancake thickness, Doble et al. (2015) find a highly variable attenuation rate, ranging from $10^{-6}$ to $10^{-3}$ over a two-week period for the frequency range used here. Their measurements suggest that $k_{i}$ values on the order of $10^{-5}$ or less are found only with pancake thicknesses under $10 \mathrm{~cm}$. For 20-cm-thick pancakes like those used in the present model simulations, Doble et al. (2015) find attenuation rates roughly between $10^{-4}$ and $10^{-3}$. Rogers et al. (2016) note that their pancake thicknesses varied between 5 and $45 \mathrm{~cm}$, but details are not provided on the distribution of thicknesses over the course of the measurements. While a closer match to observed conditions would be desirable for these simulations, the presently available datasets do not include specific details about ice floe thickness, spacing, and open water fraction concurrent with the wave time series measurements. Although the wave-ice system is not presently configured to simultaneously model frazil and pancake ice, we anticipate that such a capability will be added in the near future, at which point these field results will be reexamined.

The coupled wave-ice system described here is still under development and imposes limitations on floe shapes, sizes, and scales. Relative scaling requirements between the wave and ice models place limitations on how much each model's scales may be adjusted. Roughly speaking, the individual ice elements in a floe must be small enough that at least five contiguous elements can fit along one dimension of a single fluid cell. If not, the designations of "ice filled" cells can vary too irregularly over time and may cause instability in the wave model. Individual ice floes must be large enough that a single floe covers an area corresponding to at least $5 \times 5$ fluid cells, so that pressure variations from cell to cell do not produce excessive stress gradients in the ice floe. Floes must be at least two elements thick, but four or more element layers are recommended to better capture internal stress and strain distributions. To accurately represent higher-frequency waves, the fluid cells need to be small relative to the wavelengths employed in the simulations. Increasing the scale of wavelengths, on the other hand, would also require an increase in water depth (to preserve deep-water conditions), accompanied by a larger number of vertical $\sigma$ levels, as well as an increase in ice floe sizes to maintain relative scales.

In the multifloe cases mentioned above [sections 3c(1) and $3 c(2)$ ], a reduction of grid spacing in the wave model would require a similar reduction in the scale of the ice floe elements. If the floe sizes were kept constant, then this would entail, for example, an eightfold increase in the total number of elements for every $50 \%$ reduction in element diameter. Because the ice model element interactions are the most computationally expensive part of the wave--ice model, this would also mean a nearly eightfold increase in required computing time. In the reflection and transmission cases of section $3 \mathrm{c}(1)$, for example, such a $50 \%$ scale reduction would increase computing time for each simulation to over one week (using 64 cores).

Additional features that are currently being incorporated into the coupled system include wave overtopping of ice floes, collisions among floes, and temperature variations with associated ice melting and growth. The long-term objective is to create a highly realistic MIZ simulation platform, governed by the basic physics of waves and ice, that can be applied to far more complex 3D scenarios. The system will provide an inexpensive and convenient alternative or supplement to costly and complex field experiments.

Acknowledgments. We thank the two anonymous reviewers, whose comments and suggestions have helped to significantly improve this paper. This work has been funded by the Office of Naval Research Program Element 0601153N and Award N0014-14-0569. Modeling work was made possible through a grant of supercomputer time from the Department of Defense High Performance Computing Modernization Program (HPCMP) at the U.S. Army Engineer Research and Development Center (ERDC), as a part of Subproject NRLSS06632054. Supporting data may be obtained by contacting the corresponding author.

\section{REFERENCES}

Bateman, S., F. Shi, M. Orzech, J. Veeramony, and J. Calantoni, 2014: Discrete element method simulations of ice floe dynamics. 2014 Fall Meeting, San Francisco, CA, Amer. Geophys. Union, Abstract EP43C-3580.

, M. Orzech, F. Shi, J. Veeramony, and J. Calantoni, 2016: Simulating sea ice floes using the discrete element method. 2016 Ocean Sciences Meeting, New Orleans, LA, Amer. Geophys. Union, Abstract HE24A-1426. 
Bennetts, L. G., M. A. Peter, V. A. Squire, and M. H. Meylan, 2010: A three-dimensional model of wave attenuation in the marginal ice zone. J. Geophys. Res., 115, C12043, https://doi.org/ 10.1029/2009JC005982.

, A. Alberello, M. H. Meylan, C. Cavaliere, A. V. Babanin, and A. Toffoli, 2015: An idealised experimental model of ocean surface wave transmission by an ice floe. Ocean Modell., 96, 85-92, https://doi.org/10.1016/j.ocemod.2015.03.001.

Chung, D. H., 2008: Water oscillation in an open tube. ISB J. Phys., 2 (1), http://isjos.org/JoP/vol2iss1/Papers/JoPv2i12TubeOscill.pdf.

Derakhti, M., J. T. Kirby, F. Shi, and G. Ma, 2015: NHWAVE: Model revisions and tests of wave breaking in shallow and deep water. University of Delaware Center for Applied Coastal Research Research Rep. CACR-15-18, $127 \mathrm{pp}$.

Doble, M., G. DeCarolis, M. Meylan, J. Bidlot, and P. Wadhams, 2015: Relating wave attenuation to pancake ice thickness, using field measurements and model results. Geophys. Res. Lett., 42, 4473-4481, https://doi.org/10.1002/ 2015 GL063628.

Dumont, D., A. Kohout, and L. Bertino, 2011: A wave-based model for the marginal ice zone including a floe breaking parameterization. J. Geophys. Res., 116, C04001, https:// doi.org/10.1029/2010JC006682.

Herman, A., 2013: Numerical modeling of force and contact networks in fragmented sea ice. Ann. Glaciol., 54, 114-120, https://doi.org/10.3189/2013AoG62A055.

_ 2017: Wave-induced stress and breaking of sea ice in a coupled hydrodynamic discrete-element wave-ice model. Cryosphere., 11, 2711-2725, https://doi.org/10.5194/tc-112711-2017.

Hopkins, M. A., and H. H. Shen, 2001: Simulation of pancake ice in a wave field. Ann. Glaciol., 33, 355-360, https://doi.org/ 10.3189/172756401781818527.

— , and A. S. Thorndike, 2006: Floe formation in arctic sea ice. J. Geophys. Res., 111, C11S23, https://doi.org/10.1029/ 2005JC003352.

Keller, J. B., 1998: Gravity waves on ice-covered water. J. Geophys. Res., 103, 7663-7669, https://doi.org/10.1029/97JC02966.

Kloss, C., C. Goniva, G. Hager, S. Amberger, and S. Pirker, 2012: Models, algorithms and validation for opensource DEM and CFD-DEM. Progress Comp. Fluid Dyn., 12, 140-152, https:// doi.org/10.1504/PCFD.2012.047457.

Kohout, A. L. and M. H. Meylan, 2008: An elastic plate model for wave attenuation and ice floe breaking in the marginal ice zone. J. Geophys. Res., 113, C09016, https://doi.org/10.1029/ 2007JC004434.

—_, M. J. M. Williams, S. M. Dean, and M. H. Meylan, 2014: Storm-induced sea-ice breakup and the implications for ice extent. Nature, 509, 604-607, https://doi.org/10.1038/ nature 13262.

Liu, A. K., P. W. Vachon, C. Y. Peng, and A. S. Bhogal, 1992: Wave attenuation in the marginal ice zone during LIMEX. Atmos.-Ocean, 30, 192-206, https://doi.org/10.1080/ 07055900.1992 .9649437$.

— S. Hakkinen, and C. Y. Peng, 1993: Wave effects on ocean-ice interaction in the marginal ice-zone. J. Geophys. Res., 98, $10025-10036$, https://doi.org/10.1029/93JC00653.

Ma, G., F. Shhi, and J. T. Kirby, 2012: Shock-capturing nonhydrostatic model for fully dispersive surface wave processes. Ocean Modell., 43-44, 22-35, https://doi.org/10.1016/ j.ocemod.2011.12.002.
_ A. A. Farahani, J. T. Kirby, and F. Shi, 2016: Modeling wavestructure interactions by an immersed boundary method in a $\sigma$-coordinate model. Ocean Eng., 125, 238-247, https://doi.org/ 10.1016/j.oceaneng.2016.08.027.

Meylan, M. H., L. G. Bennetts, and A. L. Kohout, 2014: In situ measurements and analysis of ocean waves in the Antarctic marginal ice zone. Geophys. Res. Lett., 41, 5046-5051, https:// doi.org/10.1002/2014GL060809.

Montiel, F., V. A. Squire, and L. G. Bennetts, 2016: Attenuation and directional spreading of ocean wave spectra in the marginal ice zone. J. Fluid Mech., 790, 492-522, https://doi.org/ 10.1017/jfm.2016.21.

Orzech, M., F. Shi, J. Calantoni, S. Bateman, and J. Veeramony, 2014: Small-scale modeling of waves and floes in the marginal ice zone. 2014 Fall Meeting, San Francisco, CA, Amer. Geophys. Union, Abstract C11A-0347.

_, J. Veeramony, S. Bateman, J. Calantoni, and J. Kirby, 2016a: Incorporating floating surface objects into a fully dispersive surface wave model. Ocean Modell., 102, 14-26, https://doi.org/10.1016/j.ocemod.2016.04.007.

_ J. Veeeramony, F. Shi, S. Bateman, and J. Calantoni, 2016b: Modeling small-scale physics of waves and ice in the MIZ. 2016 Ocean Sciences Meeting, New Orleans, LA, Amer. Geophys. Union, Abstract A24B-2571.

Peter, M. A., and M. H. Meylan, 2010: Water-wave scattering by vast fields of bodies. SIAM J. Appl. Math., 70, 1567-1586, https://doi.org/10.1137/090750305.

Plimpton, S., 1995: Fast parallel algorithms for short-range molecular dynamics. J. Comput. Phys., 117, 1-19, https://doi.org/ 10.1006/jcph.1995.1039.

Polojärvi, A., and J. Tuhkuri, 2013: On modeling cohesive ridge keel punch through tests with a combined finite-discrete element method. Cold Reg. Sci. Technol., 85, 191-205, https:// doi.org/10.1016/j.coldregions.2012.09.013.

Rogers, W. E., J. Thomson, H. H. Shen, M. J. Doble, P. Wadhams, and S. Cheng, 2016: Dissipation of wind waves by pancake and frazil ice in the autumn Beaufort Sea. J. Geophys. Res. Oceans, 121, 7991-8007, https://doi.org/ 10.1002/2016JC012251.

Song, A., B. F. Morriss, E. J. Deeb, J. Richter-Menge, D. K. Perovich, and M. A. Hopkins, 2014: High-resolution sea ice dynamics modeling using the discrete element method. AGU 2014 Fall Meeting, San Francisco, CA, Amer. Geophys. Union, Abstract C54A-08.

Squire, V. A., J. P. Dugan, P. Wadhams, P. J. Rottier, and A. K. Liu, 1995: Of ocean waves and sea ice. Annu. Rev. Fluid Mech., 27, 115-168, https://doi.org/10.1146/annurev.fl.27.010195.000555.

Thomson, J., 2015: ONR sea state DRI cruise report, R/V Sikuliaq, fall 2015. APL-UW Tech. Rep. SKQ201512S, 45 pp., http:// www.apl.washington.edu/project/projects/arctic_sea_state/pdfs/ cruise_report.pdf.

— Ocean. Geophys. Res. Lett., 41, 3136-3140, https://doi.org/ 10.1002/2014GL059983. , and Coauthors, 2013: Sea state and boundary layer physics of the emerging Arctic Ocean. APL-UW Tech. Rep. TR1306, 59 pp.

Timco, G. W., and W. F. Weeks, 2010: A review of the engineering properties of sea ice. Cold Reg. Sci. Technol., 60, 107-129, https://doi.org/10.1016/j.coldregions.2009.10.003.

Toffoli, A., L. Bennetts, M. Meylan, C. Cavaliere, A. Alberello, J. Elsnab, and J. Monty, 2015: Sea ice floes dissipate the energy of steep ocean waves. Geophys. Res. Lett., 42, 8547-8554, https://doi.org/10.1002/2015GL065937. 
Tolman, H. L., 1997: User manual and system documentation of WAVEWATCH-III version 1.15. NOAA/NWS/NCEP/MMAB Tech. Note 151, 97 pp.

Wadhams, P., 1986: The seasonal ice zone. The Geophysics of Sea Ice, N. Untersteiner, Ed., Plenum Press, 825-991. , and B. Holt, 1991: Waves in frazil and pancake ice and their detection in Seasat synthetic aperture radar imagery. J. Geophys. Res., 96, 8835-8852, https://doi.org/10.1029/ 91JC00457.
Wang, R. and H. H. Shen, 2010: Gravity waves propagating into an ice-covered ocean: A viscoelastic model. J. Geophys. Res., 115, C06024, https://doi.org/10.1029/2009JC005591.

Xu, Z., A. Tartakovsky, and W. Pan, 2012: Discrete-element model for the interaction between ocean waves and sea ice. Phys. Rev., 85E, 016703, https://doi.org/10.1103/PhysRevE.85.016703.

Zhao, X., and H. Shen, 2015: Ocean wave transmission and reflection by viscoelastic ice covers. Ocean Modell., 92, 1-10, https://doi.org/10.1016/j.ocemod.2015.05.003. 